\title{
ArcheoSciences
}

Revue d'archéométrie

41-1 | 2017

Varia

\section{Interprétations sociales des transferts de grandes lames polies en jades alpins dans l'Europe néolithique}

Analyses spatiales dans le cadre du programme ANR JADE 2

Social interpretations of the transfers of Alpine jades axe-heads in the Neolithic Europe. Spatial analysis in the framework of the ANR Program JADE 2

\section{Estelle Gauthier et Pierre Pétrequin}

\section{OpenEdition}

Journals

Édition électronique

URL : https://journals.openedition.org/archeosciences/4856

DOI : 10.4000/archeosciences.4856

ISSN : 2104-3728

Éditeur

Presses universitaires de Rennes

Édition imprimée

Date de publication : 21 juin 2017

Pagination : 7-23

ISBN : 978-2-7535-5890-8

ISSN : 1960-1360

Référence électronique

Estelle Gauthier et Pierre Pétrequin, «Interprétations sociales des transferts de grandes lames polies en jades alpins dans l'Europe néolithique », ArcheoSciences [En ligne], 41-1 | 2017, mis en ligne le 21 juin 2019, consulté le 28 janvier 2022. URL : http://journals.openedition.org/archeosciences/4856 ; DOI : https://doi.org/10.4000/archeosciences.4856 


\title{
Interprétations sociales des transferts de grandes lames polies en jades alpins dans l'Europe néolithique Analyses spatiales dans le cadre du programme ANR JADE 2
}

\author{
Social Interpretations of the Transfers of Alpine Jades Axe-heads \\ in the Neolithic Europe \\ Spatial Analysis in the Framework of the ANR Program JADE 2
}

\author{
Estelle Gauthier ${ }^{a}$ et Pierre PÉtrequin ${ }^{b}$
}

\begin{abstract}
Résumé : Le programme ANR JADE 2 est consacré à l'étude des longues haches et des anneaux-disques en jades alpins (jadéitites, omphacitites et éclogites à grain très fin) en Europe pendant les $V^{e}$ et IV $V^{e}$ millénaires av. J.-C. Tirés des gîtes des Alpes italiennes, les objets en jade ont circulé à travers toute l'Europe occidentale, sur des distances considérables atteignant $2000 \mathrm{~km}$ à vol d'oiseau. Derrière l'apparente unité de ces réseaux de transfert, à partir de centres de production en Piémont, en Ligurie et en Émilie occidentale, se dessinent des modes de diffusion complexes, impliquant des sélections des matières premières et des transformations des objets. Un inventaire systématique et détaillé à l'échelle de toute l'Europe, exploité sous la forme d'analyses spatiales, permet à présent de détailler quelques mécanismes à l'origine de cet exceptionnel phénomène de transferts. L'image qui se dégage des répartitions et des contextes de dépôt est celle de sociétés fortement inégalitaires, où les élites contrôlaient la circulation des jades les plus valorisés pour l'affichage social et les rituels religieux. La multiplicité des réseaux de transferts, des phénomènes de sélection visant une augmentation de la valeur ajoutée, des réappropriations régionales des plus belles pièces afin d'en relancer la valeur idéelle, toutes ces particularités prouvent que les transferts des haches en jades alpins sont liés à des processus de diffusion particulièrement complexes, où les facteurs sociaux, politiques et culturels prenaient le pas sur les fonctionnements économiques.
\end{abstract}

\begin{abstract}
The ANR program JADE 2 is dedicated to the study of long axeheads and ring-discs made of Alpine jades (jadeitites, omphacitites and finegrained eclogites) in Europe, during the $V^{\text {th }}$ - $V^{\text {th }}$ millennia BC. Exploited at high-altitude quarries in the Italian Alps, jade objects circulated throughout western Europe, over considerable distances, up to 2,000 km. Beyond the apparent unity of these transfer networks, from the centers of production in Piedmont, Liguria and western Emilia, a complex way of diffusion appears, involving selections of raw material and transformations of objects. A systematic and detailed inventory across Europe exploited with a set of spatial analysis makes possible now to understand some of the mechanisms behind this unique phenomenon of transfers. The picture drawn by the distributions and contexts of burying is highly unequal societies in which the elite controlled the circulation of the most valued jades for their social representation and religious rituals. The multiplicity of transfer networks, selection processes aiming to increase their added value, regional re-appropriations of the best pieces in order to revive their ideational value, are particular phenomena which prove that the transfers of Alpine jades axeheads are related to particularly complex diffusion processes where the social, politic and cultural factors took precedence over the economic functions.
\end{abstract}

Mots clés : analyse spatiale, facteurs sociaux, jades alpins, hache polie, Néolithique, transferts.

Keywords: Alpine jades, axe-heads, Neolithic, social factors, spatial analysis, transfers.

a Université de Franche-Comté, CNRS UMR 6249 Chrono-Environnement, 30-32 rue Mégevand, 25030 BESANÇON cedex. (estelle.gauthier@univfomte.fr)

${ }^{b}$ MSHE C.N. Ledoux, CNRS et Université de Franche-Comté, 32 rue Mégevand, 25030 BESANÇON. (archeo.petrequin@free.fr) 


\section{INTRODUCTION}

Le programme ANR JADE $2^{1}$ (2013-2017, E. Gauthier et P. Pétrequin [dir.], MSHE C.N. Ledoux) est consacré à l'étude des productions en jades alpins qui ont fait l'objet d'une large diffusion en Europe au cours du Néolithique. À partir de la fin du $\mathrm{VI}^{e}$ millénaire et jusqu'à la fin du IVe millénaire av. notre ère, les objets produits dans le nordouest de l'Italie ont circulé dans toute l'Europe sur environ $2000 \mathrm{~km}$ à vol d'oiseau. Pour l'essentiel, leur répartition concerne l'Europe occidentale, mais certaines haches en jades alpins sont parvenues jusqu'à la Mer Noire. Les travaux publiés sur le sujet ont depuis longtemps démontré la valeur sociale de ces objets qui étaient de véritables signes-sociaux à valeur idéelle (Pétrequin et al., 2003; 2006a; 2012a; 2013).

Après une présentation du programme JADE 2 et des méthodes de travail, nous traiterons plus spécifiquement des résultats de l'analyse spatiale. Nous souhaitons en effet comprendre les modes de diffusion des longues haches en jades en tenant compte des facteurs sociaux et économiques qui ont influencé les transferts. Loin de refléter une répartition homogène, la circulation des jades présente en effet une grande variabilité dans l'espace. Il s'agit donc pour nous d'identifier les diverses tendances de leur diffusion et de les comparer.

\section{LE PROGRAMME JADE 2}

\section{Objectifs du programme}

Les productions en jades alpins sont principalement des lames de hache ou d'herminette et des anneaux-disques en jadéitite, omphacitite ou éclogite à grain fin. Ces matières premières ont été exploitées dans les Alpes italiennes, plus précisément dans le massif du Mont Viso près de Turin, entre 1500 et $2400 \mathrm{~m}$ d'altitude, et dans celui du Mont Beigua au nord de Gênes. Le programme JADE 2 a pour objectif de poursuivre l'étude des sources de matière première afin de mieux connaitre les modes d'exploitation et la diffusion des jades, mais aussi les mécanismes sociaux qui ont sous-tendu leur production et leurs transferts.

JADE 2 fait suite à un premier programme ANR, JADE (2006-2010, P. Pétrequin [dir.]; Pétrequin et al., 2011; Pétrequin, Cassen et al., 2012a); cette première approche a non seulement permis de réaliser un inventaire des longues haches en jades, pratiquement exhaustif dans la plus grande partie de l'Europe occidentale, mais a aussi permis des

1. programme ANR-12-BSH3-0005. avancées majeures sur les exploitations alpines (Pétrequin, Errera et al., 2006; Pétrequin, Pétrequin et al., 2007), les procédés de mise en forme (Pétrequin, Pétrequin et al., 2008; Pétrequin, Errera et al., 2009) et les réseaux de diffusion (Pétrequin, Errera et al., 2012a; Pétrequin, Cassen et al., 2012b). Le projet JADE s'est également attaché aux modalités techniques et sociales d'exploitation des jades (des expéditions saisonnières en montagne, où intervenaient des rituels et des spécialisations techniques privilégiant les meilleures matières premières; une production épisodique, augmentant la rareté de ces objets ostentatoires [Pétrequin et al., 2008; 2011]).

Les principaux objectifs du Projet JADE2 (Pétrequin, Sheridan et al., 2015) sont d'une part de poursuivre le repérage et l'étude des exploitations alpines, d'autre part de compléter l'inventaire des jades, notamment dans la péninsule ibérique et dans le sud de l'Italie - des zones jusque-là peu documentées - mais aussi et surtout, en Europe centrale et sud-orientale afin de mieux percevoir les limites du phénomène dans l'aire de l'Europe dite " du cuivre ", par opposition à l'Europe " du jade ». Il s'agit aussi de comprendre les modes de diffusion des jades alpins, et d'étudier d'autres productions impliquées dans leurs transferts : des objets accompagnant leur diffusion, des circulations en retour ou encore des imitations locales. Au cœur de nos préoccupations se trouvent aussi les mécanismes sociaux qui ont favorisé la diffusion des produits en jades alpins à travers des sociétés et des cultures très différentes, ce qui témoigne de leur valeur considérable et de leur importante force de pénétration (Cassen et al., 2010; 2012a; Klassen et al., 2011; Pétrequin, Cassen et al., 2009; 2012a). Une approche ethnologique des contextes de découverte doit de plus mettre en valeur le rôle de ces produits socialement valorisés dans la représentation et le marquage social (Pétrequin et al., 2013).

\section{Organisation du programme Jade2 et méthodes de travail}

Notre projet est fondé sur le repérage systématique des haches (notamment celles mesurant plus de $13,5 \mathrm{~cm}$, Pétrequin, Cassen et al., 2012b) et des anneaux en jades alpins (Pétrequin, Cassen et al., 2015a; 2015b) dans toutes les collections archéologiques européennes entre l'Atlantique et la Mer Noire. Léloignement progressif des zones d'étude doit permettre d'identifier les limites ultimes de la circulation des jades en direction de l'est, tout en continuant en parallèle le travail de fond sur les aires de production en Italie. La constitution d'un réseau international de spécialistes répartis le plus largement possible dans cette immense aire géographique s'avère donc essentielle (Antonović, 
2008; Bernabò Brea et al., 2012; Biró, 2009; Cassen et al., 2010; Chiarenza et Giustetto, 2010; Fábregas et al., 2012; Klassen et al., 2009; Occhi, 2008; Pailler, 2009; Rodríguez Rellán et Fábregas Valcarce, 2011; Sheridan et al., 2010; Trnka, 2010; Tsonev, 2008; Vaquer et al., 2011; Venturino Gambari, 1996). Il s'agit en effet de favoriser, dans toutes les régions concernées, la recherche efficace des objets potentiellement en jades alpins avant de les analyser pour déterminer les types de roches utilisées et leurs sources les plus plausibles. Les collaborateurs du programme ont en charge, chacun dans sa région, de répertorier les lames polies en jades alpins (Klassen 2012; Pétrequin, 2012; Pétrequin, Errera $e t$ al., 2012b; Fábregas Valcarce et al., 2012; Fábregas Valcarce et Rodríguez-Rellán, 2015; Mazzieri et Occhi, 2011-2013), d'établir les contacts nécessaires pour accéder aux collections muséographiques, d'associer leurs collègues néolithiciens proches pour étudier le contexte de découverte de chaque hache et enfin de suggérer de premières interprétations en fonction des spécificités culturelles régionales (Bendö et al., 2014, Dominguez-Bella et al., 2016).

En Italie, mais aussi dans les Grisons et en Valais, ainsi que dans les Pyrénées (afin d'identifier les sources de roche de la famille des néphrites; Vaquer et Pétrequin 2015), le repérage des gîtes sur le terrain se poursuit, ainsi que la collecte d'échantillons qui alimentent un vaste référentiel sur lequel s'appuient les déterminations. Les échantillons collectés font l'objet d'analyses pétrographiques et minéralogiques (lames minces, microscope polarisant, microsonde, diffractométrie de rayons-X, susceptibilité magnétique) par A. Prichystal à l'Université de Brno (République Tchèque, Prichystal et Trnka, 2001; Prichystal, 2013) qui servent notamment à étalonner les analyses spectroradiométriques en réflectance diffuse réalisées sur les objets archéologiques afin de déterminer la nature des roches et leur gîte d'origine lorsque c'est possible (voir ci-dessous fig. 2a). Cette méthode mise au point par Michel Errera (Musée royal de l'Afrique centrale, Tervuren, Belgique, Errera et al., 2006) a de nombreux avantages : rapide et totalement non destructive, elle peut être réalisée directement dans les musées (Errera et al., 2012).

Outre l'étude détaillée des contextes de découverte, il s'agit également de comprendre comment les jades alpins ont été intégrés au répertoire iconographique néolithique, particulièrement en contexte carnacéen. Serge Cassen (UMR 6566, Nantes) et son équipe étudient l'intégration des lames polies et des anneaux en jades dans le système des signes carnacéens, dont l'influence a été profonde en Europe occidentale (Cassen, 2012). À cette fin, ils mettent en œuvre sur des gravures et stèles du Morbihan, du Bassin parisien, de Bourgogne et de Suisse romande un protocole combinant la photogrammétrie, la photographie sous éclairages tournants et la lasergrammétrie pour créer des modèles $3 \mathrm{D}$ d'une très grande précision (Cassen et al., 2013; 2014b).

À l'opposé de ces diffusions occidentales de modèles et d'idées, nous souhaitons aussi comprendre les premières interactions entre l'Europe sud-orientale du cuivre et de l'or et l'Europe occidentale du jade (Boric, 2009; Higham et al., 2008; Klassen et al., 2012a; Pétrequin et Vaquer, 2015). Samuel van Willigen (Musée national suisse, Zurich), quant à lui, analyse des objets en cuivre susceptibles d'être issus d'une métallurgie précoce en Italie, liée probablement aux influences balkaniques et à la circulation des jades en direction de la Mer Noire pendant le $V^{e}$ millénaire. La méthode choisie est l'ablation laser adaptée à un système portatif (LA ICP MS), les analyses des échantillons étant ensuite réalisés à Zurich.

Enfin un autre volet méthodologique vise à comprendre en détail les modes de transferts et les mécanismes qui ont sous-tendu la diffusion des produits en jades (Pétrequin, Cassen et al., 2012b). Cet aspect est supporté à la fois par des modèles ethnoarchéologiques de Nouvelle-Guinée et par une analyse cartographique prenant en compte à la fois les principaux caractères des lames polies et leur chronologie; c'est ce mode d'étude spatio-temporelle que nous allons développer ici.

\section{AnAlyse SPatiale de la Diffusion DES HACHES EN JADES ALPINS}

\section{Objectifs et méthodes}

Les haches en jades alpins sont des biens socialement valorisés communs à nombre de cultures néolithiques d'Europe occidentale (Cassen et al., 2012); cependant, à partir d'une large aire de production en Italie du Nord, leurs répartitions sont loin d'être uniformes à l'échelle européenne. Un de nos objectifs est donc de rendre compte de la variabilité de la distribution des longues haches en jade (Pétrequin et al., 1997; 1998; Pétrequin, Cassen et al., 2012b). L'analyse cartographique doit en effet prendre en considération la nature différente des objets étudiés afin de distinguer des particularités chronologiques ou régionales (Renfrew, 2005). Les critères choisis sont les matières premières (en privilégiant le système d'opposition prévalent durant le $V^{\mathrm{E}}$ millénaire : roches sombres - éclogites et omphacitites versus roches claires - jadéitites et certaines omphacitites - Pétrequin, Sheridan et al., 2011, Coccato et al., 2013) et l'origine (massif du Mont Viso versus massif du Mont Beigua, Pétrequin, Errera et al., 2012c), l'appartenance typologique (Pétrequin, Sheridan et al., 2008; Pétrequin, Cassen et al., 2012b), le degré de finition, la lon- 
gueur des pièces complètes (Pétrequin, Cassen et al., 2012b) et le contexte de découverte (Cassen et al., 2012). Les répartitions doivent être comparées selon ces multiples critères et, dans la mesure du possible, en les combinant. Bien qu'une simple cartographie des répartitions soit suffisamment efficace à elle seule pour montrer les phénomènes spatiaux particuliers à ces différents descripteurs, une modélisation des points moyens accompagnés de leurs ellipses de déviation standard (Hodder et Orton, 1976 : 207-208; Pumain et Saint-Julien, 1997 : 54) fera davantage ressortir les phénomènes spatiaux (pour des exemples en archéologie : Bertoncello et al., 2012; Gauthier, 2004; Nuninger et al., 2012; Poirier, 2010), et permettra en outre, de comparer plus efficacement plusieurs distributions sur une même carte (Pumain et Saint-Julien, 2001 : 166). Bien entendu il faut se souvenir que cette méthode simple permet de créer un point qui n'a qu'une valeur statistique et présente seulement une tendance très générale. Ainsi un point moyen situé plutôt au centre de la distribution, avec une ellipse de déviation standard large et circulaire, indique une distribution vaste et relativement uniforme. Au contraire, un point moyen éloigné du centre avec une ellipse de taille réduite correspond à une distribution régionalisée, et si l'ellipse est ovalaire, la distribution tend à privilégier une direction en particulier. Il n'en demeure pas moins que la méthode des points moyens (bien qu'elle lisse les détails) est un bon outil de visualisation des tendances et de contrôle de l'interprétation des cartes de répartition.

Des analyses de distance permettront ensuite de tester des modèles théoriques de diffusion, couramment utilisés en Archéologie, impliquant différentes formes de décroissance en fonction de la distance (Samzun et al., 2012; Pétrequin, Gauthier et al., 2012 : 545). La projection des données sur un axe permettra en outre une représentation plus à même d'étudier les voies de diffusion. Les méthodes d'analyse spatiale à mettre en œuvre ont été discutées dans le cadre du programme ANR ArchaeDyn II (2009-2012, F. Favory et L. Nuninger [dir.], MSHE C.N. Ledoux, Gandini et al., 2012), plus précisément au sein d'un atelier dédié à la modélisation des dynamiques spatiales liées à la production, à la consommation ou aux transferts de matières premières et de produits manufacturés (Gauthier et al., 2013). Par souci de lisibilité, la comparaison de différents critères (types) sera faite sur des courbes de tendances, afin de pouvoir les comparer plus aisément.

\section{Résultats et interprétations}

Sans évoquer pour l'instant les exemplaires connus en Europe centrale et sud-orientale, la répartition des longues haches en jades alpins couvre toute l'Europe occidentale (figure 1), de la péninsule ibérique jusqu’au Danemark. Une proportion importante de découvertes s'observe à proximité immédiate des gîtes et encore dans le midi de la France, ainsi que le long des vallées du Pô, du Rhône, de la Seine et du Rhin. Cela s'explique par la position privilégiée de ces régions dans les circulations, tandis que les vides ou les effectifs plus faibles s'expliquent par leur position plus marginale ou plus éloignée des axes de circulation principaux.

On doit naturellement se poser la question d'éventuels biais documentaires dans les régions où les grandes lames polies sont rares ou absentes. Une carte dite de "fiabilité " (Nuninger et al., 2012; Ostir et al., 2008) a été réalisée pour traduire l'état des connaissances dans la zone étudiée (Pétrequin, Cassen et al., 2012b); d'une manière générale, la répartition des longues haches en jades en Europe est jugée fiable et représentative de tendances réelles pour le Néolithique. De surcroît, pendant le projet Jade2, les régions considérées comme pauvres en grandes lames ont été beaucoup plus intensément explorées que les autres : le nombre des artefacts y a parfois été légèrement augmenté, mais sans pour autant compenser les "déficits " déjà reconnus.

Ainsi, en tenant compte des effets conjugués de la position dans les réseaux et d'éventuels biais documentaires (le seul flagrant étant le front de la Première Guerre mondiale), on peut dire qu'à l'échelle de l'Europe occidentale l'espace est relativement bien couvert et que les plus faibles effectifs aux périphéries peuvent naturellement s'expliquer par une décroissance induite par la distance.

\section{Des phénomènes de sélection}

Néanmoins une concentration très importante se remarque dans la région de Carnac en Morbihan du fait de la découverte de nombreuses haches dans des monuments funéraires ou des dépôts. Cette concentration semble traduire un véritable phénomène d'attraction de ce secteur en contradiction avec une simple diffusion de proche en proche. Il en va de même, si l'on regarde la distribution dans ses détails, pour plusieurs autres concentrations remarquables de découvertes (autour des lacs de Neuchâtel et Genève, en Auvergne, en Aquitaine, autour de Toulouse et de Barcelone, dans le Chalonnais, le sud du Bassin parisien, en Seine-Maritime, en Hesse et Thuringe, en Écosse, etc.), à distance des gîtes, qui ne peuvent simplement s'expliquer par l'accessibilité le long des réseaux de transferts. Ces accumulations autorisent par ailleurs à distinguer des grands ensembles régionaux (Pétrequin et al., 2003, figure 3). On voit donc que la répartition des jades est loin d'être uniforme, tant par la répartition des sites de découverte que par les effectifs. On peut aller plus loin dans l'interprétation des données. 


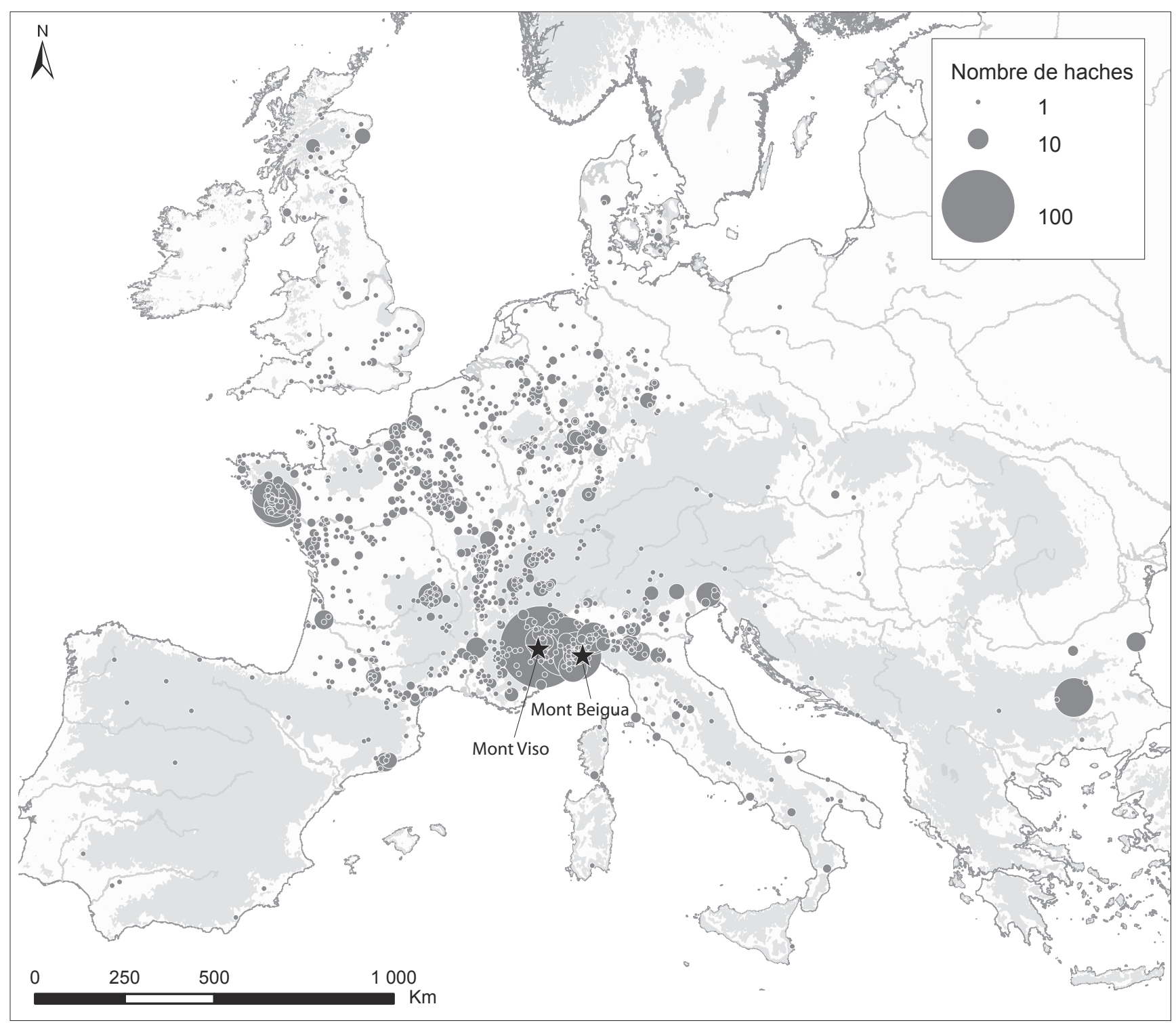

Figure 1 : Répartition générale des longues haches en jades alpins (Données : JADE 2014, P. Pétrequin. Fond : ESRI WBM, NASA SRTM. CAO : E. Gauthier, 2016).

Figure 1: General distribution of long Alpine jade axeheads (Data: JADE 2014, P. Pétrequin, ESRI WBM, NASA SRTM. Cartography: E. Gauthier, 2016).

La prise en compte de différents descripteurs doit en effet permettre de mieux comprendre les mécanismes de la diffusion en faisant apparaître notamment des différences régionales. On constate tout d'abord que la distinction des critères " origine " (déterminée par comparaison entre les analyses des lames polies et celle des échantillons naturels de notre référentiel européen) et "famille de matériaux " laissent apparaître un double système d'opposition à petite échelle. En ce qui concerne l'origine (figure 2a), l'opposition est orientée est-ouest : les productions du massif du mont Viso se retrouvent dans toute l'Europe occidentale, tandis que l'Europe sud-orientale aurait plutôt reçu celles du massif du Mont Beigua, diffusées en direction de la mer Noire un peu avant le milieu du Ve millénaire. Des connexions spécifiques des réseaux de transferts sont sans doute à l'origine de cette préférence (Errera et al., 2006; Pétrequin, Cassen et al., 2012b). 


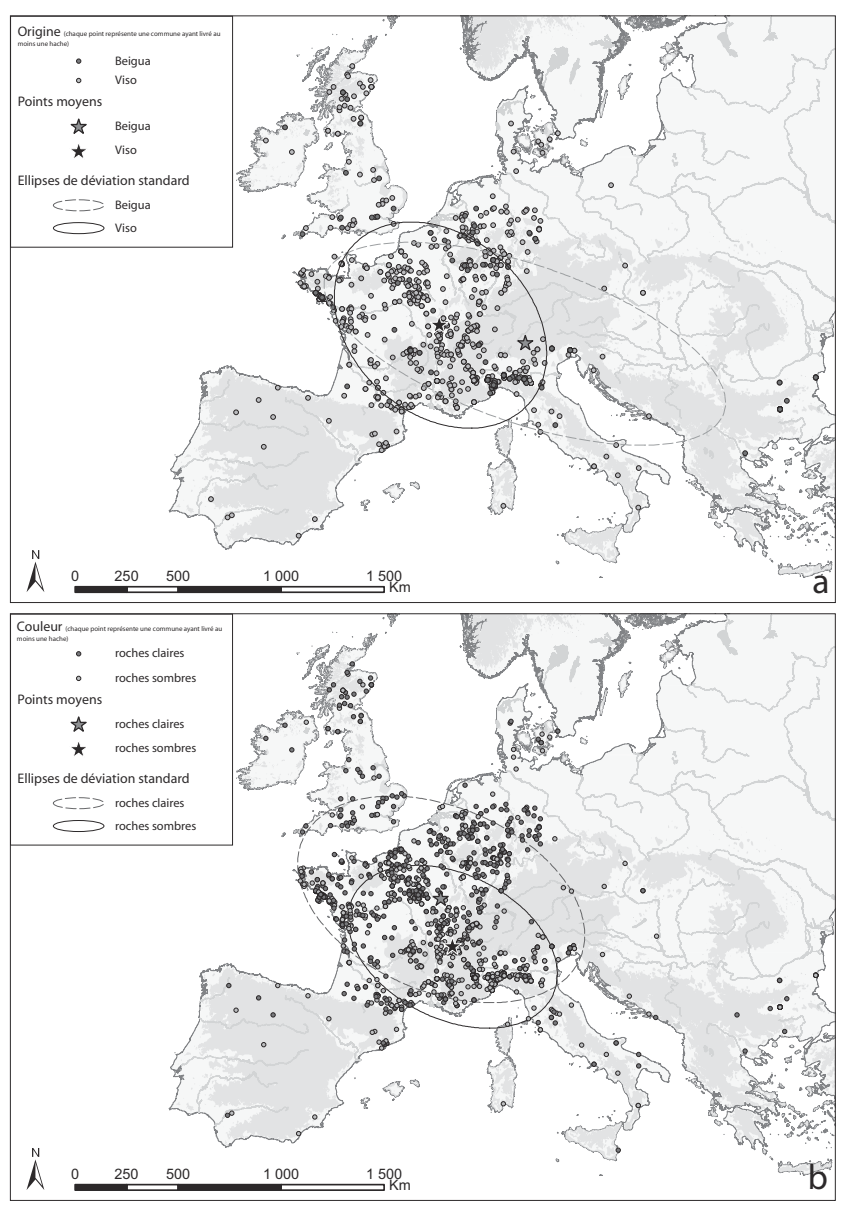

Figure 2 : (Voir planche couleur I) La différenciation de la distribution des longues haches en jades alpins selon l'origine de la matière première $(\mathrm{a})$ et la couleur des roches (b) fait apparaître un double système d'opposition en Europe (Données : JADE 2014, P. Pétrequin. Fond : ESRI WBM, NASA SRTM. CAO : E. Gauthier, 2016).

Figure 2: (See colour plate I) The differentiation of the distribution of long Alpine jade axeheads according to the origin of the raw material (a) and the colour of rocks (b) shows a double opposition system in Europe (Data: JADE 2014, P. Pétrequin, ESRI WBM, NASA SRTM. CAC: E. Gauthier, 2016).

Pour les choix de couleur des matières premières, un caractère essentiel au Néolithique, au contraire, l'opposition est orientée nord-sud (figure 2b, Pétrequin et al., 2011) : les roches claires (jadéitites en majorité) se rencontrent surtout dans la partie septentrionale du domaine de diffusion des jades alpins (Bassin parisien, Allemagne, Grande Bretagne, etc.) et les roches sombres (omphacitites, éclogites, certaines jadéitites mixtes) dans la zone méridionale (midi de la France, Italie du Nord, Espagne).

Mais si l'on parle des roches elles-mêmes, l'essentiel des artefacts en éclogite se concentre près des carrières, avec une diminution rapide, suivie d'un transfert régulier et en faible nombre. Au contraire, les jadéitites et les omphacitites jadéitiques sont peu représentées dans les exploitations et montrent une concentration remarquable entre 650 et $1000 \mathrm{~km}$, en particulier vers le milieu du $\mathrm{V}^{\mathrm{e}}$ millénaire, les préférences pouvant varier selon le type de lame polie (Pétrequin, Cassen et al., 2012b). L'idée d'un mode de diffusion différent entre éclogites et jadéitites vient immédiatement à l'esprit. Mais ce n'est probablement pas le meilleur - et en tout cas pas le seul - système explicatif. Les sondages dans les carrières du mont Viso / Bulè en septembre 2007 (Pétrequin, Pétrequin et al., 2007) ont montré que la jadéitite était globalement plus rare et probablement plus précieuse que l'éclogite dans les gîtes primaires eux-mêmes (Pétrequin et al., 2011). La valorisation sociale de la jadéitite était déjà telle, sur les sources de matière première, que même les plus petites ébauches étaient redescendues en vallée, tandis que des ébauches de hache en éclogite de $15 \mathrm{~cm}$ de longueur ou parfois davantage étaient abandonnées sur les postes de taille si elles n'étaient pas parfaites. L'hypothèse de deux modes de diffusion n'est probablement pas fausse ici, mais reste à un niveau très superficiel des systèmes explicatifs, tandis que la valorisation sociale différente de l'un et l'autre groupes de roches permet de mieux comprendre la gestion différente des ébauches et des outils. Il est apparu de plus que les deux groupes de carrières du mont Viso, l'un surtout avec de l'éclogite, l'autre surtout avec de la jadéitite, avaient été probablement exploitées par deux groupes humains et culturels différents (Pétrequin, Pétrequin à paraître).

On se doute, bien sûr, qu'au cours du millénaire que compte le cycle social de circulation des haches en jades, ces objets valorisés ont subi des variations de forme, phénomène classique de l'affichage des inégalités sociales dans un système d'objets-signe (Chancerel et al., 2015). Le programme JADE a établi une typo-chronologie des longues haches en jades (Pétrequin, Cassen et al., 2012b) que nous employons ici. Alors que certains types ont une diffusion large (Puy, Durrington, Chelles), d'autres ont une diffusion bien plus limitée (Bégude à proximité des gîtes alpins, Saint-Michel et Tumiac en Bretagne, Pauilhac dans le midi de la France). Des types régionaux se distinguent (figure 3a) : certains peuvent être qualifiés de "méridionaux " (Bégude, Pauilhac), car ils sont surtout présents en Italie et dans le sud de la France; d'autres sont dits "septentrionaux » (Altenstadt-Greenlaw, Glastonburry et Chenoise), car ils se retrouvent surtout du Bassin parisien à la Grande-Bretagne et au Danemark, avec une concentration importante en Allemagne; enfin certains sont nommés " carnacéens " (Bernon, Tumiac, Saint-Michel), car ils sont pour l'essentiel concentrés dans le Morbihan. Si 


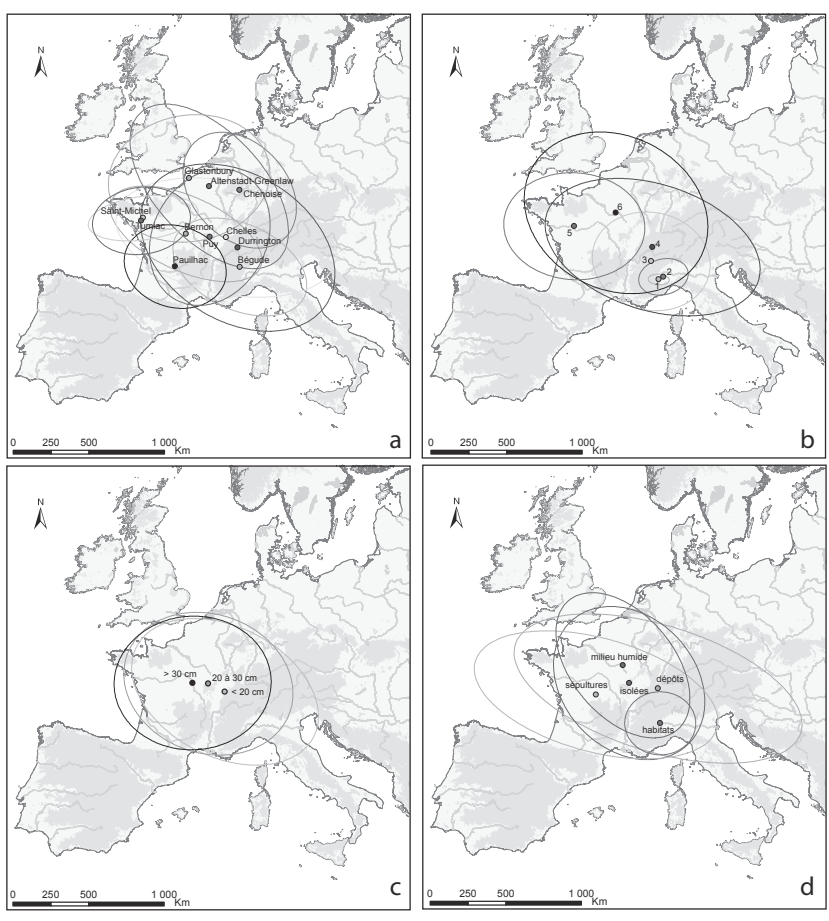

Figure 3 : (Voir planche couleur II) Comparaison, à l'aide des points moyens et des ellipses de déviation standard, des tendances principales de la distribution des haches en jades alpins distinguées selon différents critères, la typologie (a), le niveau de finition (b), la longueur (c) et le contexte de découverte (d) (Données : JADE 2014, P. Pétrequin. Fond : ESRI WBM, NASA SRTM. CAO : E. Gauthier, 2016).

Figure 3: (See colour plate II) Comparison, with the help of mean centers and standard deviation ellipses, of the principal trends in the distribution of Alpine jades axeheads distinguished by different criteria, typology (a) the level of finish (b), the length (c) and the context of discovery (d) (Data: JADE 2014, P. Pétrequin, ESRI WBM, NASA SRTM. CAC: E. Gauthier, 2016).

des préférences régionales peuvent expliquer ces tendances, des raisons chronologiques sont aussi en cause : la prise en compte de la typo-chronologie montre tout d'abord une expansion progressive du phénomène vers le nord-ouest, par étapes, avec sur la fin des phases de repli (Pétrequin, Sheridan et al., 2015). Pendant le $V^{e}$ millénaire, les haches en jades ont en effet suivi la frontière entre cultures méridionales et danubiennes en direction de la Bretagne, puis l'expansion du Michelsberg depuis le Bassin parisien vers l'Allemagne à partir de 4300 av. J.-C. (Jeunesse et al. 2003), enfin la colonisation néolithique de la GrandeBretagne et de l'Irlande au début du IV $V^{e}$ millénaire (Klassen et al., 2009; Pailler et Sheridan, 2009; Pétrequin, Sheridan et al., 2008). Le type Bégude, le plus ancien, a ainsi connu une diffusion qui ne dépassait guère $800 \mathrm{~km}$ à vol d'oiseau, tandis que les types Altenstadt, Tumiac et Saint-Michel sont les plus éloignés de la zone de production au moment de la plus grande intensification de la circulation.

Pour ces objets socialement valorisés, l'équipe JADE a aussi très tôt reconnu l'importance de la plus ou moins grande régularité des formes et de la qualité de la finition et du polissage (Pétrequin, Cassen et al., 1997; 2007). Six catégories ont été ainsi distinguées pour l'étude fine des objets (Pétrequin, Cassen et al., 2012b), depuis les ébauches (niveaux 1, non polies et 2, à polissage partiel), les outils d'abattage (niveaux 3, à bouchardage et polissage partiel et 4, à polissage total irrégulier), jusqu'aux exemplaires polis à glace (niveau 5) ou même décorés ou perforés (niveau 6). La cartographie des centres de gravité des répartitions par type de polissage (figure $3 \mathrm{~b}$, les catégories ayant ici été regroupées pour accentuer le poids statistique) montre bien que, schématiquement, la qualité du polissage crôit depuis les carrières (niveaux 1 et 2) en direction de la Bretagne et du nord de l'Europe (niveaux 5 et 6). Si les ébauches se concentrent surtout au niveau des aires de production, les exemplaires qui ont subi un polissage plus intensif se retrouvent plus souvent loin des gîtes alpins. Cette augmentation est progressive depuis la zone alpine en direction du nord et de l'ouest, mais elle ne se fait pas partout à la même vitesse : lente d'abord sur les 500 premiers kilomètres, puis de façon exponentielle entre 800 et $1200 \mathrm{~km}$.

Si l'on tient compte de la longueur des pièces complètes (figure $3 \mathrm{c}$ ), on constate que les exemplaires de très grande taille, surdimensionnés par rapport à leur fonction technique, sont nettement plus fréquents dans les régions périphériques. Tant pour la longueur des pièces que pour le niveau de finition, cela peut correspondre à une sélection progressive des exemplaires ayant une plus forte valeur sociale, au fur et à mesure que l'on s'éloigne de la région source. Ils gagnent ainsi à la fois en exotisme, en ancienneté (les transferts étant relativement lents, un important décalage chronologique existe entre des objets d'un même type découverts dans le Piémont et en Grande Bretagne), en esthétisme et en valeur symbolique (les pièces les plus longues, les plus brillantes, les plus fines) pour devenir de véritables signes-sociaux.

Enfin, les contextes de découverte viennent encore appuyer l'hypothèse d'une sélection des jades selon la distance, favorisant les meilleurs marqueurs sociaux (figure $3 \mathrm{~d}$ ). Une grande majorité des haches en jades alpins ont été trouvées isolées (seules et enterrées hors de tout contexte domestique ou funéraire). La répartition de celles-ci ne montre pas de tendance spécifique, mais les autres contextes présentent des particularités remarquables : alors que les haches en jades trouvées en habitats sont majoritaires dans un cercle de $200 \mathrm{~km}$ autour des gîtes, où elles sont surtout utilisées 
comme outils d'abattage (poli 3 ou 4), au delà, elles sont plus fréquemment disposées dans des dépôts (Pétrequin, Cassen et al. 2009) et des tombes. Certaines sépultures riches et monumentales étaient selon toute vraisemblance réservées à des personnages de haut rang (Cassen et al., 2011 ; 2012a ; Pétrequin, Sheridan et al., 2008 ; RoussotLaroque, 2008), comme les tumulus carnacéens géants en Morbihan ou certaines tombes des nécropoles de Varna. Les dépositions volontaires de lames polies en jades s'accompagnent quant à elles d'une forme de ritualisation (Pétrequin, Sheridan et al., 2012), avec des positions particulières (plantées verticalement, tranchant vers le haut), le choix de contextes spécifiques liés aux montagnes, aux pierres dressées mais aussi à l'eau (tourbières, marais, bords de rivières, etc., " milieu humide » sur la figure $3 \mathrm{~d}$ ), ainsi que des dépôts symboliques par paires, ou encore des destructions volontaires (haches brisées ou brûlées). On ajoutera leur représentation sur de nombreuses stèles et parois de monuments carnacéens, que l'on retrouve par ailleurs de façon très similaire en Bourgogne et dans le Bassin parisien (Cassen, 2007; Cassen et al., 2014a). Il est très probable que les lames en jades aient été très tôt intégrées pleinement à la mythologie néolithique, en particulier carnacéenne (Cassen, 2000 ; 2012). Ainsi, à distance des sources de matières premières, les haches en jades alpins, avaient acquis une valeur nouvelle, celle d'objets-signes et appartenaient surtout à la sphère sociale et religieuse.

\section{Des centres de consommation et de redistribution}

La question des modes de diffusion est fondamentale; dans le cas de la production et de la distribution de l'obsidienne en Anatolie, elle a été abordée en détail par C. Renfrew (1975) qui a proposé les seuls modèles théoriques aujourd'hui encore utilisés. Il s'agit pour nous de reproduire cet exercice afin d'identifier parmi ces différents modes de diffusion, celui qui correspond le mieux aux lames polies d'origine alpine; pour ce faire, nous nous appuyons sur les effectifs d'objets. Nous souhaitons en particulier savoir si la diffusion des haches en jades répond ou non à un modèle "down-the-line" (de proche en proche) depuis longtemps cité comme mode de diffusion privilégié des productions néolithiques (Clark, 1955).

Nous choisissons donc de calculer la distance de chaque hache (sauf celles du Beigua) par rapport au massif du mont Viso qui est la source principale des jades alpins et est considéré comme l'origine principale des réseaux de transfert en direction du Nord-Ouest de l'Europe - et de représenter les effectifs sous forme d'histogrammes selon un pas de $10 \mathrm{~km}$. Le graphique général pour l'Europe occidentale (figure 4) présente en fait deux tendances associées : certes, une dimi- nution numérique progressive avec la distance, mais aussi de multiples pics de plus ou moins grande amplitude tout le long de l'axe, espacés de façon presque régulière tous les $100 \mathrm{~km}$ environ. On identifie ainsi de larges concentrations, séparées par des zones à faible densité de vestiges. L'hypothèse de transferts de proche en proche (Renfrew, 1975, fig. 11), ou bien encore de transferts de biens de prestige (Renfrew, 1975, fig. 14) ne peut rendre compte de cette disposition géographique très particulière, faite de concentrations régionales successives. La situation s'apparente en fait à un modèle avec intermédiaires (Renfrew, 1975, fig. 12), fonctionnant en "places centrales " installées le long des axes de diffusion principaux (Renfrew, 1975, fig. 10, $\mathrm{n}^{\circ}$ 5) et faisant office de centres de redistribution. Nous voyons ici l'effet de la circulation de biens hautement valorisés dans un système social inégalitaire où les élites sont concentrées dans des régions à forte densité de population (Pétrequin et al., 1998; 2003). Les haches auraient été redistribuées entre élites régionales qui les ont intégrées à des pratiques de thésaurisation et de destruction ritualisée, mais en ont aussi transféré certaines en direction des élites plus lointaines. C'est ainsi que les concentrations de haches les plus importantes vont se situer aux deux extrémités du réseau : chez les producteurs et leurs voisins immédiats d'une part - où la circulation s'apparente à une diffusion de proche en proche (Samzun et al., 2012) - et dans les communautés où les fonctionnements sociaux sont très inégalitaires, où elles correspondent à une thésaurisation avec une faible remise en circulation.

\section{Des fonctionnements différents selon les voies de diffusion}

Mais à vouloir traiter toute l'information géographique d'un seul coup, des biais méthodologiques apparaissent, qui ont pour effet de neutraliser les spécificités régionales. Une approche globale ne peut pas être satisfaisante dans la mesure où les chemins empruntés suivent plusieurs axes distincts et peuvent correspondre à des logiques de diffusion différentes. Il est donc nécessaire de séparer les artefacts en ensembles géographiques distincts. Cinq axes ont ainsi été définis et les analyses de distance sont réalisées tour à tour suivant chacun d'eux. Les axes 1 et 5 ont pour origine le centre des exploitations du mont Viso, l'axe 1 partant en direction du Bassin parisien et l'axe 5 suivant la voie méridionale vers le Morbihan. Les axes 2, 3 et 4 partent du centre de la plus grande concentration dans le Bassin parisien, à $500 \mathrm{~km}$ du mont Viso, l'axe 2 allant vers le nord de la France et la Grande-Bretagne, l'axe 3, vers l'Allemagne et l'axe 4 vers la Bretagne (figure 5). L’analyse est réalisée en tenant compte des mêmes critères distinctifs que précédemment, par exemple la typologie. Pour confronter plus aisément 
Figure 4 : Variation de la distance au Mont Viso des lieux de découverte des longues haches en jades alpins (hormis celles provenant du Mont Beigua) (Données : JADE 2014, P. Pétrequin. DAO : E. Gauthier, 2016).

Figure 4: Variation of the distance to Mont Viso of the find places of long Alpine jade axeheads (except those from Mont Beigua) (Data: JADE 2014, P. Pétrequin. CAD: E. Gauthier, 2016).
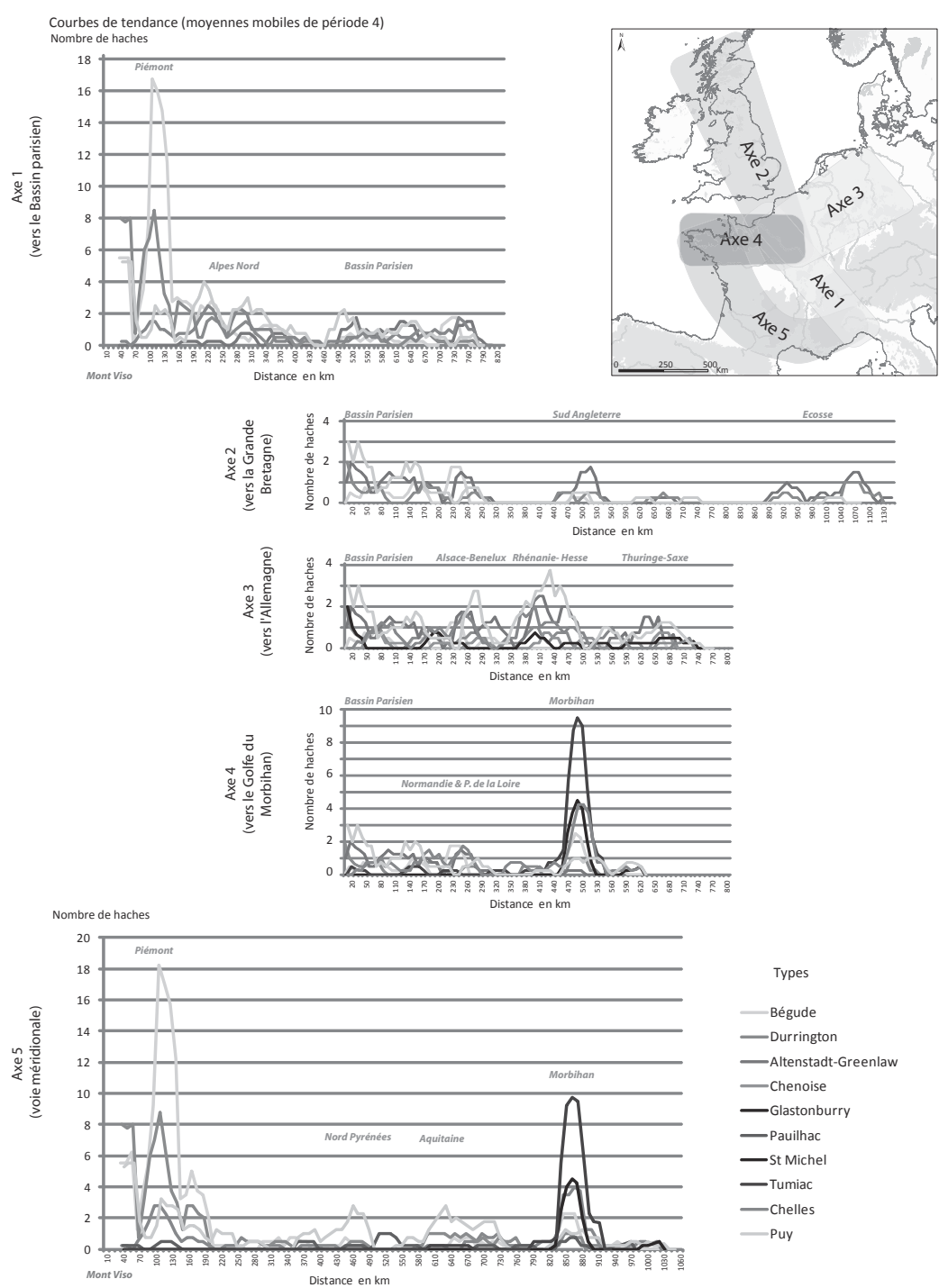

Remarques : les calculs ont été effectués en distances euclidiennes. Les effectifs sont calculés par pas de $10 \mathrm{~km}$

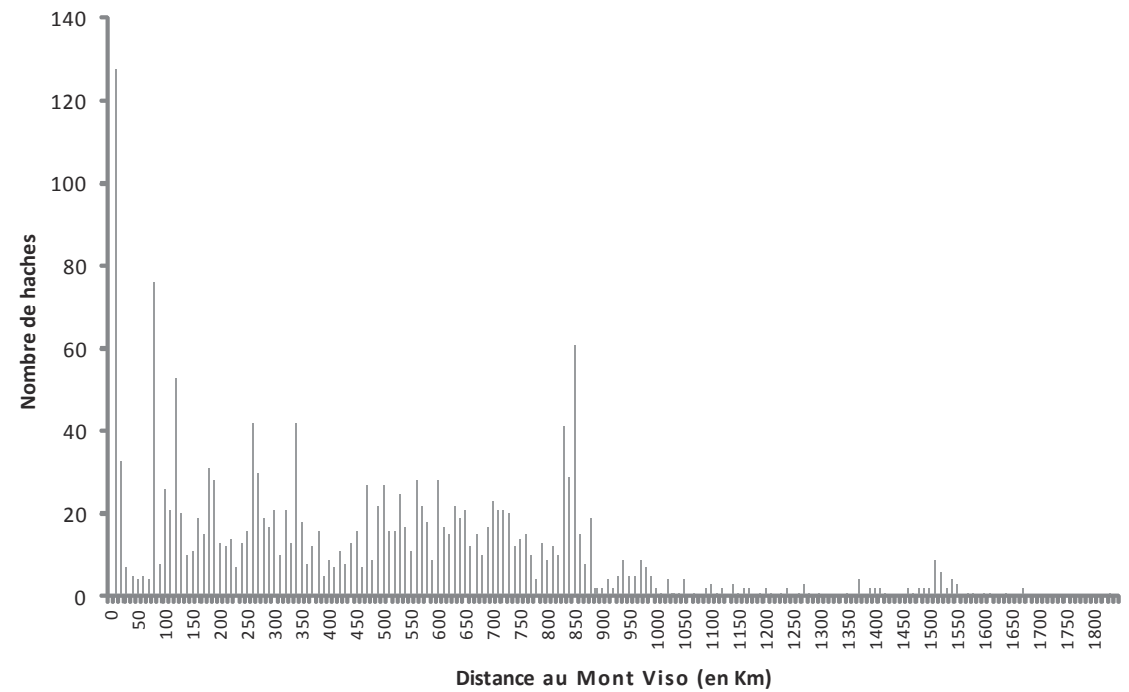

Figure 5: (Voir planche couleur III) Variation de la distance au Mont Viso des longues haches en jades alpins, distinguées selon leur type et observées le long des 5 axes définis comme les principales voies de diffusion (Données : JADE 2014, P. Pétrequin. DAO : E. Gauthier, 2016).

Figure 5: (See colour plate III) Variation of the distance to Mont Viso of long Alpine jade axeheads, distinguished according to their type and observed along the 5 axes defined as the main distribution routes (Data: JADE 2014, P. Pétrequin. CAD: E. Gauthier, 2016). 
les profils nous ne conservons des histogrammes que des courbes de tendance qui, bien qu'elles lissent quelque peu les résultats, reflètent la forme des distributions et permettent de comparer la position et l'amplitude relative des pics d'un nombre assez important de types de haches différents sur un même graphique.

Si nous prenons comme exemple l'axe 1 , nous constatons que les pics relatifs aux différents types de haches sont sensiblement les mêmes, ce qui témoigne d'une certaine stabilité des grands " centres" de consommation des haches en jades. On note tout d'abord une concentration très importante dans les cent premiers kilomètres autour du Viso. Cela correspond aux haches et ébauches trouvées dans la région piémontaise et alpine et concerne surtout les types les plus anciens, dits méridionaux, Bégude et Durrington. On en trouve en effet une quantité très importante au sein des habitats et ateliers du nord de l'Italie. Le type Bégude se fait rare après $200 \mathrm{~km}$. Le type Durrington connaît un schéma assez comparable, mais il reste assez bien représenté tout le long de l'axe 1. Le type Altenstadt-Greenlaw est quasiment absent jusqu'à $250 \mathrm{~km}$ environ. C'est surtout à partir de $500 \mathrm{~km}$, dans le Bassin parisien, que ce type connaît les concentrations les plus importantes (probablement par suite du repolissage des Durrington, dont une partie du stock en circulation a été transformé en Altenstadt). D'une façon générale, cette zone est particulièrement riche et les types représentés y sont très variés. Ce secteur semble faire office de plaque tournante dans la diffusion des haches en jades en direction des axes 2, 3 et 4, marqués chacun par des pics de types régionaux, de faible amplitude mais réguliers. L'autre grand centre d'attraction, plus marqué encore, se trouve à l'extrémité de l'axe 4, dans le Golfe du Morbihan. Là se concentrent non seulement un grand nombre d'exemplaires de haches en jades, mais également une grande diversité de types, avec en particulier deux types spécifiques à cette région, dits " carnacéens ", Tumiac et Saint-Michel.

L'axe 5, par la voie méridionale et atlantique, montre un fonctionnement très différent. Hormis deux pics très importants en début et fin de parcours, l'axe ne connaît pas de concentrations de forte amplitude. Tandis que le type Bégude connaît une décroissance rapide dès les cent premiers kilomètres, le type Pauilhac - qui se rencontre seulement sur cette voie de diffusion - ne présente que des concentrations modestes. Il n'existe donc sur cette voie pas de centre de redistribution jouant un rôle aussi important que la concentration du Bassin parisien sur l'axe septentrional.

Ces analyses mettent en évidence des fonctionnements très différents selon les types. La courbe de décroissance du nombre de haches de type Bégude ne correspond pas vraiment au modèle down-the-line; avec une raréfaction rapide des exemplaires, elle suggère plutôt des échanges interrégionaux d'un bien à forte valeur ajoutée entre les élites (Samzun et al., 2012). Le modèle va ensuite en se complexifiant; les formes se régionalisent, un type de hache chassant l'autre pour répondre à des goûts régionaux et aux besoins de la compétition sociale. Des séries de concentrations plus ou moins fortes jalonnent le parcours de ces objets et témoignent de la présence de places centrales où certaines haches ont été thésaurisées, tandis que d'autres étaient redistribuées en direction des élites plus lointaines. Enfin le golfe du Morbihan apparaît comme la zone d'accumulation ultime, le principal foyer d'attraction des jades alpins.

Plusieurs changements surviennent au moment de la mise en place de la circulation du type Puy, un type tardif qui commence à prendre de l'importance vers la fin du $V^{e}$ millénaire. Outre des décalages des aires de concentration, on observe que le type Puy marque la fin de la régionalisation et de l'opposition nord-sud encore très nette à la phase précédente. Par ailleurs, alors que les transferts de jades alpins se poursuivent, comme en témoignent les fortes concentrations sur les principaux axes de communication, la présence du type Puy est moins marquée dans les périphéries, comme en Grande-Bretagne, mais surtout en Bretagne. Ce phénomène semble correspondre à la fin de l'attractivité de ces régions, peut-être en raison de l'adoption de nouveaux modèles régionaux d'objets-signes (Grande-Bretagne) et d'une modification de la structure sociale (Morbihan) qui ne permet plus de soutenir les importations à très longue distance.

\section{Des transformations au cours des transferts}

Nous avons mis en évidence des phénomènes d'ordre spatial et chronologique qui témoignent de la complexité des réseaux de transferts des jades alpins : des sélections des objets ayant la plus forte valeur (sociale) ajoutée, une régionalisation des types, une accentuation du polissage, des usages différents des jades selon la distance aux gîtes, une diffusion qui passe par des centres de pouvoir et de redistribution, le golfe du Morbihan représentant le plus attractif et le plus important d'entre eux. La combinaison de ces observations permet d'aller encore plus loin. Nous devons ainsi souligner quelques singularités très révélatrices. La fréquence des très grandes haches et les niveaux de finition des objets augmentent avec la distance. Mais bien que ces sélections puissent répondre à des goûts régionaux ou bien à une valorisation croissante des objets avec l'éloignement, elles peuvent aussi correspondre à des modifications plus profondes de la symbolique des objets eux-mêmes au cours des transferts entre centres de pouvoir. 
En effet, les ébauches déterminables (niveaux 1 et 2 ) appartiennent surtout aux types alpins (Bégude, Durrington, Puymirol, Puy), tandis qu'on remarque l'absence (sauf rares exceptions, Pétrequin, Errera et al., 2009) des ébauches de types régionaux comme Altenstadt-Greenlaw, Chenoise, Glastonbury, Tumiac ou Saint-Michel (tableau 1). Les lames ayant servi d'outils d'abattage, de niveaux 3 ou 4 , correspondent le plus souvent à ces types alpins, bien que les haches de type Altenstadt-Greenlaw soient aussi bien représentées. Parmi les haches de niveau 5, le type Bégude est beaucoup moins abondant, contrairement aux haches de type Puy, Durrington, Altenstadt-Greenlaw; c'est également à ce niveau de finition que l'on trouve le plus souvent les haches de type Bernon, Chenoise et Glastonbury. Enfin le niveau 6 (surpolissage) est surtout représenté par les types Saint-Michel, Tumiac et Pauilhac, qui se trouvent essentiellement sous cette forme très aboutie (respectivement 71, 64 et $81 \%$, tableau 1 ).

L'absence d'ébauches de certains types, leur présence uniquement sous la forme d'objets finis - voire surpolis - semble indiquer un mode de production différent du modèle classique et attendu où l'ébauche préfigure déjà le type final. La rareté des ébauches loin des gîtes, la complémentarité de la distribution de certains types (figure 6) et la tendance à une augmentation progressive du polissage vont également dans le sens d'une transformation progressive des pièces au cours de leurs transferts. On pourrait voir dans l'absence des stades initiaux de la mise en forme de certains modèles, une retransformation de haches déjà existantes par des épisodes successifs de mise en forme et d'amincissement par polissage (Pétrequin, Cassen et al., 2012b; Pétrequin et al., 2013). Sélectionnées et repolies pour en modifier la forme, cette réappropriation des plus belles pièces devait permettre de relancer leur valeur idéelle (Pétrequin et al., 2011). L'équipe du programme JADE a en effet mis en évidence de telles transformations sur plusieurs objets : par exemple Altenstadt-Greenlaw vers Puy ou vers Tumiac, Bégude vers Bernon, Durrington vers Puy, Puy vers Chelles. Ces transformations successives débutant en Italie du Nord pour s'achever en Écosse laissent penser à l'existence de centres spécialisés situés sur les principaux axes de transfert et dans les centres régionaux de pouvoir.

Une partie des Durrington et Puymirol auraient ainsi été sélectionnées à leur arrivée dans le Bassin parisien et repolies pour produire des types Altenstadt-Greenlaw, dont certaines auraient poursuivi leur course en direction du nord-est (beaucoup finissant par être déposées) et d'autres en direction de la Bretagne où elles auraient été repolies en haches de type Tumiac, une conception spécifiquement carnacéenne, avec souvent d'autres modifications : perforation du talon, aménagement d'une arête médiane, typiques du domaine carnacéen. C'est ainsi qu'elles sont devenues des objets-
Effectifs par type et par niveau de finition

\begin{tabular}{|c|c|c|c|c|c|c|c|c|}
\hline Polissage & $\mathbf{1}$ & $\mathbf{2}$ & $\mathbf{3}$ & $\mathbf{4}$ & $\mathbf{5}$ & $\mathbf{6}$ & Ind. & Total \\
\hline Bégude & 38 & 12 & 51 & 111 & 42 & 1 & 13 & 268 \\
\hline Durrington & 47 & 8 & 28 & 52 & 81 & 4 & 8 & 228 \\
\hline Chelles & & 4 & 42 & 84 & 36 & & 5 & 171 \\
\hline $\begin{array}{c}\text { Altenstadt- } \\
\text { Greenlaw }\end{array}$ & 1 & 1 & 5 & 25 & 76 & 8 & 5 & 121 \\
\hline Chenoise & & & & 3 & 15 & 1 & & 19 \\
\hline Glastonbury & & & & 9 & 16 & 3 & 2 & 30 \\
\hline Puymirol & 2 & 6 & 4 & 7 & 23 & 7 & 2 & 51 \\
\hline Bernon & 1 & 2 & 1 & 8 & 23 & 4 & 3 & 42 \\
\hline Saint-Michel & & & 1 & 4 & 2 & 17 & & 24 \\
\hline Tumiac & & & & 3 & 12 & 39 & 7 & 61 \\
\hline Pauilhac & 1 & & & 1 & 1 & 22 & 1 & 27 \\
\hline Rarogne & & & 7 & 1 & 2 & 3 & & 13 \\
\hline Puy & 21 & 5 & 32 & 118 & 107 & 2 & 4 & 289 \\
\hline Indéterminé & 75 & 17 & 18 & 41 & 26 & 3 & 188 & 368 \\
\hline
\end{tabular}

\section{Proportions (en \%)}

\begin{tabular}{|c|c|c|c|c|c|c|c|}
\hline Polissage & $\mathbf{1}$ & $\mathbf{2}$ & $\mathbf{3}$ & $\mathbf{4}$ & $\mathbf{5}$ & $\mathbf{6}$ & Ind. \\
\hline Bégude & 14 & 4 & 19 & 41 & 16 & 0,4 & 5 \\
\hline Durrington & 21 & 4 & 12 & 23 & 36 & 2 & 4 \\
\hline Chelles & & 2 & 25 & 49 & 21 & & 3 \\
\hline $\begin{array}{c}\text { Altenstadt- } \\
\text { Greenlaw }\end{array}$ & 1 & 1 & 4 & 21 & 63 & 7 & 4 \\
\hline Chenoise & & & & 16 & 79 & 5 & \\
\hline Glastonbury & & & & 30 & 53 & 10 & 7 \\
\hline Puymirol & 4 & 12 & 8 & 14 & 45 & 14 & 4 \\
\hline Bernon & 2 & 5 & 2 & 19 & 55 & 10 & 7 \\
\hline Saint-Michel & & & 4 & 17 & 8 & 71 & \\
\hline Tumiac & & & & 5 & 20 & 64 & 11 \\
\hline Pauilhac & 4 & & & 4 & 4 & 81 & 4 \\
\hline Rarogne & & & 54 & 8 & 15 & 23 & \\
\hline Puy & 7 & 2 & 11 & 41 & 37 & 1 & 1 \\
\hline Indéterminé & 20 & 5 & 5 & 11 & 7 & 1 & 51 \\
\hline
\end{tabular}

Tableau 1 : Variation du degré de finition des longues haches en jades alpins selon leur type. À gauche, les effectifs; à droite, les pourcentages par niveau de finition (Données : JADE 2014, P. Pétrequin, DAO : E. Gauthier, 2016).

Tableau 1: Variation of finishing levels of long Alpine jade axeheads according to their type. On the left, the numbers of axeheads; on the right, the percentages by level of finishing (Data: JADE 2014, P. Pétrequin. CAD: E. Gauthier, 2016). 


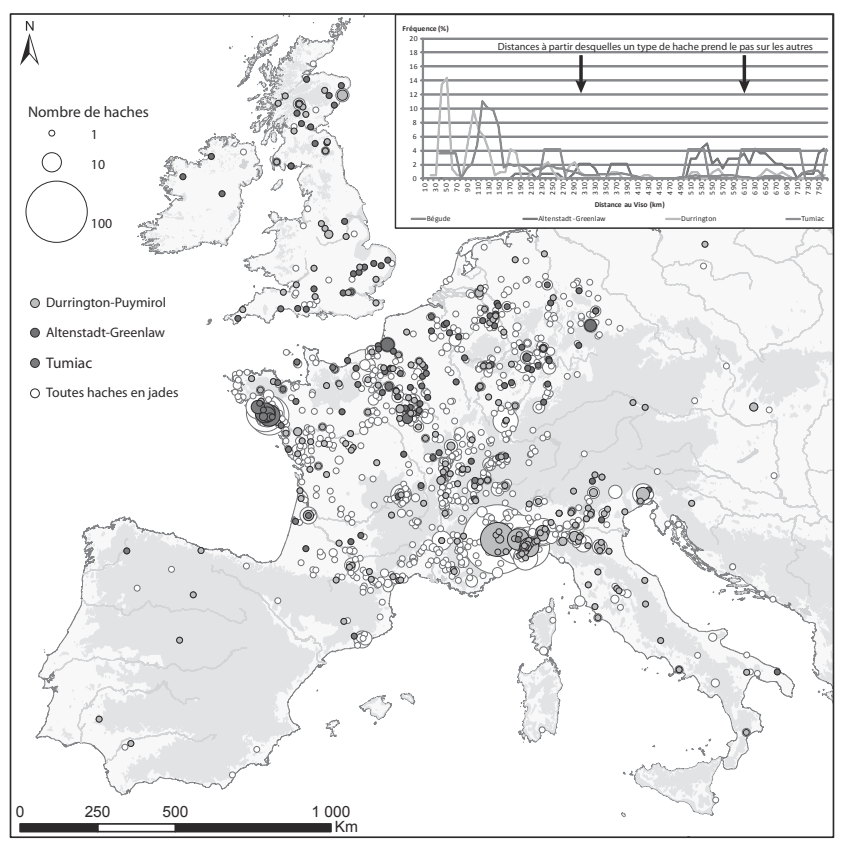

Figure 6 : (Voir planche couleur IV) Complémentarité de la distribution des types Durrington, Altenstadt et Tumiac. Cette complémentarité vient appuyer l'hypothèse de transformations par repolissage des longues haches afin de créer des types régionaux. (Données : JADE 2014, P. Pétrequin. Fond ESRI WBM, NASA SRT. CAO : E. Gauthier, 2016).

Figure 6: (See colour plate IV) Complementarity of the distribution of Durrington, Altenstadt and Tumiac types. Such a complementarity supports the hypothesis of transformations by the repolishing of long axeheads in order to create regional types (Data: JADE 2014, P. Pétrequin. CAC: E. Gauthier, 2016).

signes prestigieux ou même sacrés, manipulés par des personnages hors du commun, cumulant fonctions religieuses et fonctions politiques (Pétrequin et al., 2013).

Des phénomènes de repolissage des types Bégude et Chelles pour obtenir des lames de faible épaisseur se font aussi jour en Morbihan et participent directement à la valorisation de ces produits de l'imaginaire social. À l'autre bout de l'Europe, en Bulgarie, le même phénomène s'observe avec les types Varna (trapézoïdal) et Durrington/Chelles amincis par repolissage (Pétrequin, Cassen et al., 2012b). En Allemagne/Belgique, on a dénombré plusieurs cas de haches de type Altenstadt/Greenlaw transformées en type Puy, parfois associées à des lames en cuivre dans les dépôts. Apparaissant vers 4300 av. J.-C., ce type a été conçu sur le modèle d'outils en cuivre (Pétrequin et al., 2015), un phénomène qui démontre sans aucun doute une influence de l'Europe du cuivre (Cassen, 2003; Klassen et al., 2012a; Roussot-Laroque, 2008).

ArcheoSCiences, revue d'archéométrie, 41(1), 2017, p. 7-23

\section{Influence du modèle carnacéen et circulations en retour}

Vers le milieu du Ve millénaire, Carnac et le golfe du Morbihan semblent constituer l'épicentre de l'Europe du Jade. Le golfe du Morbihan a attiré un nombre remarquable d'objets-signes exotiques (figure 7) : les haches et les anneaux en jades alpins bien sûr, mais aussi des perles et des pendeloques en variscite (Cassen et Vaquer, 2015; Guilaine et al., 2015; Pétrequin et al., à paraître; Querré, Calligaro et al., à paraître) et probablement des haches en fibrolite de la péninsule ibérique (Pétrequin, Sheridan et al., 2015).

En retour, le succès des modèles carnacéens (et de leur signification religieuse) fut tel que des haches en jades repolies en Morbihan ont été diffusées dans toutes les directions sur $1000 \mathrm{~km}$ au moins à vol d'oiseau (figure 7, Cassen, 2003; Pétrequin, Cassen et al., 2012c), accompagnant vraisemblablement la diffusion du "mégalithisme " et d'une partie de la mythologie du golfe du Morbihan jusque dans les Alpes suisses et en Allemagne centrale (Klassen et al., 2012a; 2012b; Pétrequin, Pétrequin et al., 2007; Pétrequin, Cassen et al., 2009; Sheridan et al., 2010). On remarque en effet que le polissage de niveau 5 et les aménagements typiquement carnacéens - le talon perforé ou une arête médiane - sont présents jusqu'en Italie du Nord, ce qui correspond sans doute à un retour de haches à fort niveau de polissage en direction des aires alpines de production.

Ainsi peuvent être déterminés avec vraisemblance des axes de transfert à double sens (y compris par voie maritime), d'objets-signes caractéristiques de leurs régions d'origine et suggérant une certaine communauté d'idées (Cassen et al., 2012). Dans ce système, le Golfe du Morbihan apparaît autant comme point de départ que point d'arrivée de ces transferts d'idées et de produits socialement valorisés. Le phénomène carnacéen a d'ailleurs également suscité des imitations en roches locales (Samzun et al., 2012; Sheridan et Pailler, 2012), notamment du type Tumiac à talon perforé (Pétrequin, Cassen et al., 2012c), comme le type Cangas dans l'ouest de la péninsule ibérique ou le type Zug en Suisse et en Allemagne du Sud-Ouest (figure 7) (Pétrequin, Cassen et al., 2006; Pétrequin, Gauthier et al., 2010).

\section{La fin du mythe des sociétés néolithiques égalitaires et autarciques}

Ainsi multiplicité des réseaux de transferts, phénomènes de sélection pour accroître la valeur sociale ajoutée, préférences régionales et transformations pour répondre à un besoin de réappropriation, toutes ces particularités prouvent que les transferts des haches en jades alpins sont loin d'être simples et uniformes. Les processus de diffusion ont de plus évolué dans le temps et dans l'espace en fonction des cultures 


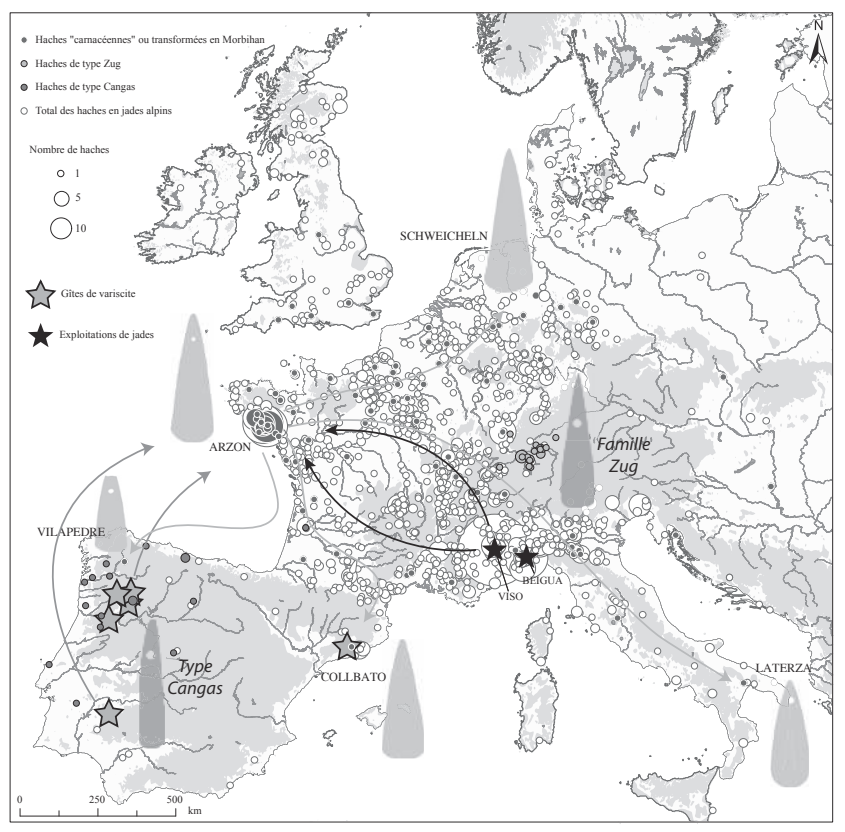

concernées ou, plus exactement selon la structure des sociétés et de leur imaginaire. La diffusion de tels produits - des outils d'abattage, devenus objets-signes sociaux et religieux dès leur sortie de gîtes de matière première - est sujette à des mécanismes complexes associant facteurs sociaux, politiques, économiques et culturels.

Le type d'étude réalisé sur les haches en jades alpins a bénéficié de conditions de recherche extrêmement favorables grâce aux travaux poussés et méticuleux, réalisés sur la très longue durée par une équipe bien organisée, celle du programme JADE. Ces travaux ont permis d'avoir des données d'une qualité remarquable, qui permettent aujourd'hui de percevoir les différentes étapes de la biographie des objets eux-mêmes. Il apparaît en effet essentiel d'appréhender les flux des jades alpins en prenant en compte les différentes étapes de parcours complexes, faits de transferts successifs, de transformations, de flux et de reflux, lors de circulations sur des distances qui se chiffrent parfois en milliers de kilomètres et pendant une durée considérable, s'étalant sur plusieurs siècles.

Un tel exemple peut sembler unique à ce jour pour la période concernée - hormis la circulation des spondyles depuis la Mer Égée ou peut-être l'Adriatique jusqu'en Bassin parisien, mais qui reste mal comprise faute d'une documentation systématique. Cependant, à notre avis, cet exemple - accompagné des transferts de variscite depuis la péninsule Ibérique - ne ferait qu'annoncer ce que l'on pressent de plus en plus clairement : l'importance, sinon la nécessité sociale, des flux transcommunautaires dans le fonctionnement des groupes néolithiques européens, circulations longtemps
Figure 7 : (Voir planche couleur IV) Principales circulations et échanges de biens socialement valorisés en Europe occidentale pendant les $V^{e}$ et $I V^{e}$ millénaires av. n.e. Le Golfe du Morbihan apparaît clairement comme l'épicentre des importations de produits socialement valorisés (haches en jades alpins, variscite espagnole). Il est autant un lieu d'attraction qu'un centre d'innovation et le point de départ d'une circulation en retour d'objets (haches carnacéennes), mais aussi de modèles (imitations locales de type Zug ou Cangas) (Données : JADE 2014, P. Pétrequin, Fond : ESRI WBM, NASA SRTM, CAO : E. Gauthier, d'après Pétrequin, Cassen et al. 2012b et Pétrequin et al. à paraître).

Figure 7: (See colour plate IV) Circulation and exchanges of socially valorzed products in Western Europe during the $5^{\text {th }}-4^{\text {th }}$ millennia BC. The Gulf of Morbihan appears clearly as the epicenter of imports of socially valorized goods (axeheads in Alpine jades and Spanish variscite). It is as much an attractive place, as a center of innovation and the starting point of a back flow of objects (carnacéen axes), but also models (local imitations of Zug and Cangas types) (Data: JADE 2014, P. Pétrequin, ESRI WBM, NASA SRTM. CAC: E. Gauthier, after Pétrequin, Cassen et al., $2012 b$ and Pétrequin et al., in press).

masquées par le mythe a-scientifique de sociétés pensées et voulues " égalitaires et autarciques " pour étayer les rêves occidentaux de l'évolutionnisme et du progrès.

\section{Remerciements}

Nous tenons à remercier tous les membres du programme $J A D E / J A D E 2$ sans lesquels cette synthèse n'aurait pas été possible, la MSHE Claude Nicolas Ledoux, en particulier Isabelle Mouret et Soizic Viaouet, pour toute l'aide administrative et logistique qu'elles nous apportent au quotidien, ainsi que l'Agence Nationale pour la Recherche qui finance ce projet, ainsi que la publication des deux prochains volumes de la monographie sur les jades alpins.

\section{Bibliographie}

Antonović D., 2008. The development of the ground stone industry in the Serbian part of the Iron Gates. In C. Bonsall, V. Boroneanţ, I. Radovanović (ed.). The Iron Gates in prehistory: new perspectives. Oxford, Archaeopress, p. 19-38 (British Archaeological Reports, International Series, 1893).

Bendő Zs., Szakmány Gy., Kasztovszky Zs., Maróti B., SzIlágyi Sz., Szilágyi V., T. Biró K., 2014. Results of non-destructive SEM-EDX and PGAA analyses of Jade and Eclogite polished stone tools in Hungary. Archeometriai Mühely, XI (4), p. 187-205.

Bernabo Brea M., Errera M., Mazzieri P., Occhi S., PÉtrequin P., 2012. Les haches alpines dans la culture des 
VBQ en Émilie occidentale : contexte, typologie, chronologie et origine des matières premières. In P. Pétrequin, Cassen et al., 2012a, p. 822-871.

Bertoncello F., Fovet E., Gandini C., Trément F., Nuninger L., 2012. Spatio-temporal dynamics of settlement patterns in central and southern Gaul from $800 \mathrm{BC}$ to $800 \mathrm{AD}$ : models for long-term interregional comparison. In Gandini et al., 2012, p. 51-64.

Bıró T. K., 2009. Lithic Raw Materials in Hungary: a diachronic presentation on recent advances in Hungarian petroarchaeology. In J. Gancarski (ed.). Surowce naturalne w Karpatach Polskich oraz ich wykorzystanie $w$ pradziejach i wczesnym średniowieczu. Muzeum Podkarpackie w Krośnie, Krosno, p. 23-40.

Boric D., 2009. Absolute dating of metallurgical innovations in the Vinča culture of the Balkans. In T. L. Kienlin, B. W. Roberts (ed.). Metals and Societies. Studies in honour of Barbara S. Ottaway. Bonn, Rudolf Habelt, p. 191-245.

Cassen S., 2000. Architecture du tombeau, équipement mortuaire, décor céramique et art gravé du V millénaire en Morbihan. A la recherche d'une cosmogonie des premières sociétés agricoles de l'Europe occidentale. In Actas do $3^{\circ}$ Congresso de Arqueologia Peninsular. Vila Real 1999, vol. IV, Pré-historia recente da Peninsula ibérica. ADECAP, Porto, p. 447-479.

Cassen S., 2003. Importer, Imiter, Inspirer? Objets-signes centreeuropéens dans le Néolithique armoricain. L'Anthropologie, 107 , p. $255-270$.

Cassen S., 2007. Un pour tous, tous contre un... Symboles, mythe et histoire à travers une stèle morbihannaise du Ve millénaire. In A. Testart, L. Barray, P. Brun (ed.). Pratiques funéraires et sociétés. Nouvelles approches en archéologie et en anthropologie sociale. Dijon, Éditions universitaires de Dijon, p. 37-67.

Cassen S., 2012. L’objet possédé, sa représentation, mise en contexte général avec stèles et gravures. In Pétrequin, Cassen et al. 2012a, p. 1310-1353.

Cassen S., Boujot C., Dominguez Bella S., Guiavarc'h M., Le Pennec C., Prieto Martinez M.P., Querre G., Santrot M.E., Vigier E., 2012. Dépôts bretons, tumulus carnacéens et circulations à longue distance. In Pétrequin, Cassen et al., 2012a, p. 918-995.

Cassen S., Boujot C., Errera M., Menier D., Pailler Y., Pétrequin P., Marguerie D., Veyrat E., Vigier E., Poirier S., Dagneau C., Degez D., Lorho T., Neveu-Derotrie H., Obeltz C., Scalliet F., Sparfel Y., 2010. Un dépôt sousmarin de lames polies néolithiques en jadéitite et sillimanite, et un ouvrage de stèles submergé sur la plage dite du Petit Rohu près Saint-Pierre-Quiberon (Morbihan). Bulletin de la Société préhistorique française, 107 (1), p. 53-84.

Cassen S., Lescop L., Grimaud V., 2013. Pour une critique de la représentation tridimensionnelle des architectures mégali- thiques en Europe occidentale. Méthodes et usages actuels. Annales de Bretagne et des pays de l'Ouest, 120, p. 7-35.

Cassen S., Lescop L., Grimaud V., Caldwell D., 2014a. A discovery of exceptional Neolithic engravings in Buthiers, Seineet-Marne, France. Antiquity: A Review of World Archaeology, 88 (340). [http://journal.antiquity.ac.uk/projgall/cassen340] (consulté en avril 2016).

Cassen S., Lescop L., Grimaud V., Robin G., 2014 b. Complementarity of acquisition techniques for the documentation of Neolithic engravings: lasergrammetric and photographic recording in Gavrinis passage tomb (Brittany, France). Journal of Archaeological Science, 45, p. 126-140.

Cassen S., Pétrequin P., Boujot C., Domínguez-Bella S., Guiavarc'H M., Querré G., 2011. Measuring distinction in the megalithic architecture of the Carnac region: from sign to material. In M. Furholt, F. Lüth, J. Müller (ed.). Megaliths and identities. Bonn, Rudolf Habelt, p. 225-248.

Cassen S., Vaquer J., 2015. La variscite ibérique. In Chancerel et al. 2015, p. 64-69.

Chancerel A., Vaquer J., Clayet-Merle J.-J., 2015. Signes de Richesse, Inégalités au Néolithique. Paris, Réunion des Musées nationaux.

Chiarenza N., Giustetto R. (ed.), 2010. Lofficina litica di Pertus (Paesana - CN): testimonianze di lavorazione e analisi minero-petrografiche. Quaderni della Soprintendenza archeologica del Piemonte, 24, p. 13-29.

Clark J.G.D., 1955. L'Europe préhistorique. Les fondements de son économie. Paris, Payot.

Coccato A., Karampelas S., Wörle M., Van Willigen S., Pétrequin P., 2013. Gem quality and archeological green "jadeite jade" vs "omphacite jade": A multi-method study. Journal of Raman Spectroscopy, 45 (11-12), p. 1260-1265.

Dominguez-Bella S., Cassen S., Petrequin P., Prichystal A., Martinez R., Ramos Munoz J., Medina N., 2016. Aroche (Huelva, Andalucía): a new Neolithic axehead of Alpine jade in the southwest of Iberian Peninsula. Journal of Field Archaeology, 8 (1), p. 205-222.

Errera M., Hauzeur A., Pétrequin P., Tsonev T., 2006. Etude spectroradiométrique d'une lame de hache trouvée dans le district de Chirpan (Bulgarie). Interdisciplinary Studies, XIX, p. 7-24.

Errera M, Pétrequin P., Pétrequin A.M., 2012. Spectroradiométrie, référentiel naturel et étude de la diffusion des haches alpines. In Pétrequin, Cassen et al., 2012a, p. 440-533.

Fábregas Valcarce R., Rodríguez-Rellán C., 2015. Walking on the stones of years. Some remarks on the NW Iberian rock art. In P. Skoglund, J. Ling (ed.). Picturing the Bronze Age. Oxford/Philadelphia, Oxbow Books, p. 47-63.

Fábregas Valcarce R., De Lombera Hermida A., RodríguezRellán C., 2012. Spain and Portugal: long chisels and per- 
forated axes. Their context and distribution. In Pétrequin, Cassen et al., 2012a, p. 1108-1135.

Gandini C., Favory F., Nuninger L. (ed.), 2012. Archaedyn. 7 millennia of territorial dynamics. Settlements pattern, production and trades from Neolithic to Middle Ages. Archaeopress, Oxford (British Archaeological Reports, International Series, 2370).

Gauthier E., 2004. L'évolution de la consommation du métal à l'Âge du Bronze, en France orientale et en Transdanubie. Histoire et Mesure, XIX (3-4), p. 345-376.

Gauthier E., Weller O., Giraud J., Brigand R., Pétrequin P., Gabillot M., 2013. Theoretical space-time modelling of the diffusion of raw materials and manufactured objects. In G. Earl, T. Sly, A. Chrysanthi et al. (ed.). E-Papers from the 40th Conference on Computer Applications and Quantitative Methods in Archaeology, Southampton, 26-30 March 2012. Amsterdam University Press, Amsteradam, 863-873 (Archaeology in the Digital Era, II).

Guilaine J., Vaquer J., Gratuze B., Querre G., 2015. Les perles en variscite de la grotte de las Claousos IV et de la grotte René Carrié. In J. Guilaine, J. Vaquer, J. Zammit (dir.). Grottes sépulcrales des Hautes Corbières. Les grottes de Las Claousos à Auriac et de René Carrié à Termes (Aude). Monographie des Archives d'Écologie Préhistorique, Toulouse, EHESS, p. 89-104.

Higham T., Chapman J., Slavchev V., Gaydarska B., Honch N., Yordanov Y., Dimitrov A., 2008. New AMS radiocarbon dates for the Varna Eneolithic cemetery, Bulgarian Black Sea Coast. Acta Musei Varnaensis, 6, p. 95-114.

Hodder I., Orton C., 1976. Spatial Analysis in Archaeology. Cambridge, Cambridge University Press.

Jeunesse C., Lefranc P., Denaire A., 2003. Groupe de Bischheim, origine du Michelsberg, genèse du groupe d'Entzheim. La transition entre le Néolithique moyen et le Néolithique récent dans les régions rhénanes. APRAA, Zimmersheim.

KLASSEN L., 2012. Axes of Alpine jade from southern Scandinavia and northernmost Germany. Danish Journal of Archaeology, 1 (1), p. 86-89.

Klassen L., Cassen S., Pétrequin P., 2012a. Alpine axes and early metallurgy. In Pétrequin, Cassen et al., 2012a, p. 12801309.

Klassen L., Dobes M., Pétrequin P., 2012b. Dreieckige Kupferflachbeile aus Mitteldeutschland und Böhmen. Zum kulturgeschichtlichen Hintergrund einer bemerkenswerten Fundgruppe, Alt-Thüringen, 41, p. 7-35.

Klassen L., Pétrequin P., Cassen S., 2011. The power of attraction... Zur Akkumulation sozial wertbesetzer alpiner Artefakte im Morbihan und im westlichen Ostseeraum im 5. und 4. Jahrtausend V.Chr. In S. Hansen, J. Müller (ed.). Sozialarchäologische Perspektiven: Gesellschaftlicher Wandel 5000-1500 v.Chr. zwischen Kaukasus und Atlantik. P. von Zabern, Darmstadt, p. 13-40.
Klassen L., Pétrequin P., Errera M., 2009. Ein herausragendes neolithisches Jadebeil aus Hiddenhausen-Bermbeck. Archäologie in Westfalen-Lippe, p. 162-165.

Mazzieri P., Occhi S., 2011-2013. Le asce nei corredi sepolcrali dell'Emilia occidentale. Rivista di Studi Liguri, LXXVIILXXIX, p. 331-338.

Nuninger L., Saligny L., Oštir K., Poirier N., Fovet E., Gandini C., Gauthier E., Kokalj Ž., Tolle F., 2012. Chapter 1- Models and tools for territorial dynamic studies. In Gandini et. al. 2012, p. 23-37.

Occhi S., 2008. Le asce levigata della collezione storica del Museo Archeologico Nazionale di Parma. Tesi di laurea in Preistoria e Protostoria, Universita degli Studi, Bologna.

Ostir K., Kokalj Z., Saligny L., Tolle F., Nuninger L., 2008. Confidence maps: a tool to evaluate archaeological data's relevance in spatial analysis. In A. Posluschny, K. Lambers, I. Herzog (ed.). Layers of perception. Bonn, Rudolf Habelt, p. 272-277 (Kolloquien zur Vor- und Frühgeschichte, 10).

Pailler Y., 2009. Neolithic Fibrolite Working in the West of France. In B. O'Connor, G. Cooney, J. Chapman (ed.). Materialitas: Working Stone, Carving Identity. Oxford, Oxbow Books, p. 113-126.

Pailler Y., Sheridan A., 2009. Everything you always wanted to know about... la néolithisation de la Grande Bretagne et de l'Irlande. Bulletin de la Société préhistorique française, 106 (1), p. 1-32.

Pétrequin P., 2012. Axes of alpine jade in Denmark: the point of view of an alpine prehistorian. Danish Journal of Archaeology, 1 (1), p. 90-91.

Pétrequin P., Cassen S., Chevillot C., Cornen G., Denaire A., Duteil Y., Pailler Y., Prodéo F., Villes A., 2015a. Bracelets en schiste et anneaux-disques en jadéitite, en serpentinite ou en amphibolite. In Chancerel et al., 2015, p. 35-42.

Pétrequin P., Cassen S., Chevillot C., Errera M., Pailler Y., Pétrequin A.M., Prichystal A., Prodéo F., 2015b. La production des anneaux-disques alpins pendant les $\mathrm{VI}^{\mathrm{e}}$ et $\mathrm{V}^{\mathrm{e}}$ millénaires av. J.-C. et le Mont Viso. In P.J. Rey, A. Dumont (dir.). L'homme et son environnement : des lacs, des montagnes et des rivières. Bulles d'archéologie offertes à André Marguet. Dijon, SAE, p. 259-302.

Pétrequin P., Cassen S., Croutsch C., 2006. Imitation ou convergence : les haches néolithiques à talon perforé au nordouest des Alpes. In L. Baray (ed.). Artisanats, sociétés et civilisations. Hommage à Jean-Paul Thévenot. Dijon, RAE, p. 163-177.

Pétrequin P., Cassen S., Croutsch C., Weller O., 1997. Haches alpines et haches carnacéennes dans l'Europe du Ve millénaire. Notae Praehistoricae, 17, p. 135-150.

Pétrequin P., Cassen S., Errera M., Gauthier E., Klassen L., Pailler Y., Pétrequin A. M., Sheridan A., 2009. L'Unique, la Paire, les Multiples. À propos des dépôts de haches polies en

ArcheoSciences, revue d'archéométrie, 41(1), 2017, p. 7-23 
roches alpines en Europe occidentale pendant les $V^{e}$ et IV ${ }^{e}$ millénaires. In S. Bonnardin, C. Hamon, M. Lauwers, B. Quilliec (ed.). Du matériel au spirituel. Réalités archéologiques et historiques des "dépôts " de la Préhistoire à nos jours. Juan-les-Pins, APDCA, p. 417-427.

Pétrequin P., Cassen S., Errera M., Gauthier E., Pétrequin A. M., Rossi G., 2007. Du Mont Viso au golfe de Tarente à la transition V-IV e millénaires : la hache en jadéitite de Laterza (Puglia, Italie). Bericht der Römisch-Germanischen Kommission, 54 (1), p. 25-52.

Pétrequin P., Cassen S., Errera M., Klassen L., Pétrequin A. M., Sheridan A., 2013. The value of things: the production and circulation of Alpine jade axes during the $5^{\text {th }}-4^{\text {th }}$ millenia in a European perspective. In T. Kerig, A. Zimmermann (ed.). Economic archaeology: from structure to performance in European archaeology. Bonn, Rudolf Habelt, p. 65-82.

Pétrequin P., Cassen S., Errera M., Klassen L., Sheridan A., PÉtrequin A.M. (dir.), 2012a. JADE. Inégalités sociales et espace européen au Néolithique : la circulation des grandes haches en jades alpins. Besançon, Presses Universitaires de FrancheComté.

Pétrequin P., Cassen S., Gauthier E., Klassen L., Pailler Y., Sheridan A., 2012b. Typologie, chronologie et répartition des grandes haches alpines en Europe occidentale. In Pétrequin, Cassen et al., 2012a, p. 574-727.

Pétrequin P., Cassen S., Klassen L., Fábregas Valcarce R., 2012c. La circulation des haches carnacéennes en Europe occidentale. In Pétrequin, Cassen et al., 2012a, p. 1015-1045.

Pétrequin P., Croutsch C., Cassen S., 1998. À propos du dépôt de La Bégude : haches alpines et haches carnacéennes pendant le $\mathrm{V}^{\mathrm{e}}$ millénaire. Bulletin de la Société préhistorique française, 95 (2), p. 239-254.

Pétrequin P., Errera M., Cassen S., Croutsch C., 2003. De la pétrographie aux approches sociales : la circulation des grandes haches en roches alpines pendant le Néolithique. In Les matières premières lithiques en préhistoire. Les Eyzies-deTayac, Cressensac, p. 253-275.

Pétrequin P., Errera M., Cassen S., Gauthier E., Klassen L., Pétrequin A. M., Sheridan A., 2012a. Austausch auf europäischer Ebene - alpine Jade des 6. bis 4. Jahrtausends v. Chr. Archäologie in Deutschland, 2012 (2), p. 22-25.

Pétrequin P., Errera M., Martin A., Fábregas Valcarce R., Vaquer J., 2012b. Les haches en jades alpins pendant les $V^{e}$ et IV millénaires. L'exemple de l'Espagne et du Portugal dans une perspective européenne. In Xarxes al neolític. Circulació i intercanvi de matèries, productes i idees a la Mediterránia occidental (VII-III millenni aC). Gavà, Museu de Gavà, p. 213222 (Rubricatum, 5).

Pétrequin P., Errera M., Pétrequin A.M., Allard P., 2006. The Neolithic Quarries of Mont Viso (Piedmont, Italy). Initial
Radiocarbon Dates. European Journal of Archaeology, 9 (1), p. 7-30.

Pétrequin P., Errera M., Pétrequin A. M., Gauthier E., 2009. Une production du Mont Viso en Italie : l'ébauche de haches de Lugrin (Haute-Savoie, France). In De la Méditerranée et d'ailleurs. Mélanges offerts à Jean Guilaine. Toulouse, Archives d'Écologie Préhistorique, p. 583-595.

Pétrequin P., Errera M., Rossy M., 2012c. Viso ou Beigua : approche pétrographique du référentiel des « jades alpins ». In Pétrequin, Cassen et al. 2012a, p. 292-419.

Pétrequin P., Gauthier E., Pétrequin A.M., 2010. Les haches en silex de type Glis-Weisweil en France, en Suisse et en Allemagne du Sud-Ouest. Des imitations de haches alpines à la transition $\mathrm{V}^{\mathrm{e}}-\mathrm{IV}^{\mathrm{e}}$ millénaires. In I. Matuschik et al. (dir.). Vernetzungen, Aspekte siedlungsarchäologischer Forschung, Festschrift für Helmut Schlichtherle. Lavori, Freiburg im Breisgau, p. 237-252.

Pétrequin P., Gauthier E., Jaccottey L., Jeudy F., Maitre A., Vaquer J., 2012. Les exploitations de Réquista (Aveyron) et de Plancher-les-Mines (Haute-Saône, France). Exemples de diffusion de haches à moyenne distance. In Pétrequin, Cassen et al. 2012a, p. 544-573.

Pétrequin P., Pétrequin A. M., avec la coll. de Chiarenza N., Cinquetti M., Mancusi V., Zamagni B., Venturino M., à paraître. Chapitre 14 : À l'origine des routes du jade alpin : spécialisation régionale et premiers transferts. À paraître dans Jade tome 3, Besançon, Presses Universitaires de Franche-Comté.

Pétrequin P., Pétrequin A. M., Cinquetti Errera M., Fábregas Valcarce R., Gauthier E., Jallet F., Pailler Y., Prodéo F., Sheridan A., à paraître. Anneaux et haches en jades alpins : importations et exportations du golfe du Morbihan pendant le Ve millénaire et le début du IVe. In Actes du colloque "Callaïs", La parure en callaïs (variscite, turquoise) du Néolithique européen. Nature, origine, exploitation, circulation, utilisation. Avril 2015, Carnac.

Pétrequin P., Pétrequin A.M., Errera M., Cassen S., Croutch C., Dufraisse A., Gauthier E., Rossy M., 2007. Les carrières néolithiques du Mont Viso (Piémont, Italie). Chronologie et conditions d'exploitation. Bulletin d'Études Préhistoriques et Archéologiques Alpines, 18, p. 167-188.

Pétrequin P., Pétrequin A. M., Errera M., Jaime Riveron O., Bailly M., Gauthier E., Rossi G., 2008. Premiers épisodes de la fabrication des longues haches alpines : ramassage de galets ou choc thermique sur des blocs? Bulletin de la Société Préhistorique Française, 105 (2), p. 309-334.

Pétrequin P., Sheridan A., Cassen S., Errera M., Gauthier E., Klassen L., Le Maux N., Pailler Y., 2008. Neolithic Alpine axeheads, from the Continent to Great Britain, the Isle of Man and Ireland. Analecta Praehistorica Leidensia, 40, p. 262-279. 
Pétrequin P., Sheridan A., Cassen S., Errera M., Gauthier E., Klassen L., Le Maux N., Pailler Y., Pétrequin A. M., Rossy M., 2011. Eclogite or jadeitite: the two colours involved in the transfer of alpine axeheads in western Europe. In V. Davis, M. Edmonds (ed.). Stone Axe Studies III. Oxford, Oxbow Books, p. 55-82.

Pétrequin P., Sheridan A., Cassen S., Errera M., Klassen L., Pétrequin A.M., 2012. Consecration and sacrifice: long Alpine jade axeheads in Neolithic Europe. In B. Ramminger, H. Lasch (ed.). Hunde - Menschen - Artefakte. Gedenkschrift für Gretel Gallay. Rahden/Westf., Leidorf, p. 33-64.

Pétrequin P., Sheridan A., Gauthier E., Cassen S., Errera M., Klassen L., 2015. Projet JADE 2. 'Object-signs' and social interpretations of Alpine jade axeheads in the European Neolithic: theory and methodology. In T. Kerig, S. Shennan (ed.), Connecting Networks: Characterising Contact by Measuring Lithic Exchange in the European Neolithic. Oxford, Archaeopress, p. 83-102.

Pétrequin P., Vaquer J., 2015. Masses, sphéroïdes et haches de pierre à perforation transversale. In Chancerel et al., 2015, p. 29-34.

Poirier N., 2010. Un espace rural à la loupe. Paysage, peuplement et territoires en Berry de la préhistoire à nos jours. Tours, Presses Universitaires François-Rabelais.

Prichystal A., Trnka G., 2001. Raw materials of polished artifacts from two Lengyel sites in Lower Austria. Slovak Geological Magazine, 7 (4), p. 337-339.

Prichystal A., 2013. Lithic raw materials in prehistoric times of Eastern Central Europe. Brno, Masaryk University.

Pumain D., Saint-Julien T., 1997. L'analyse spatiale. Paris, Armand-Colin.

Pumain D., Saint-Julien T., 2001. Les interactions spatiales. Paris, Armand-Colin.

Querré G., Calligaro T., Cassen S., Dominguez-Bella S., à paraître. Long distance provenances of the callaïs adorments along Atlantic Europe from the fifth to the third millenium. In: Raw materials exploitation in Prehistory: sourcing, processing and distribution, International congress, Faro, Portugal, 10-12 March 2016.

Renfrew C., 1975. Trade as Action at a Distance: Questions of Integration and Communication. In J. Sabloff, C. LambergKarlovsky (ed.). Ancient Civilization and Trade. Albuquerque, University of New-Mexico, p. 3-59.

Renfrew C., 2005. Characterisation and Exchange theory. In C. Renfrew, P. Bahn (ed.). Achaeology the Key Concepts. London/New York, Routledge, p. 23-26.
Rodríguez Rellán C., Fábregas Valcarce R., 2011. La industria lítica en el Noroeste de la Península Ibérica durante el III y II milenios A.C. In M. Pilar Prieto Martínez, L. Salanova (ed.). Las Comunidades Campaniformes en Galicia. Cambios sociales en el III y II milenios BC en el NW de la Península Ibérica. Diputación de Pontevedra, Pontevedra, p. 249-258.

Roussot-Laroque J., 2008. La « sépulture de chef » de Pauilhac. Préhistoire du Sud-Ouest, 16 (1), p. 91-142.

Samzun A., Pétrequin P., Gauthier E., 2012. Une imitation de hache alpine type Bégude à Buthiers-Boulancourt (Seineet-Marne) au début du $\mathrm{V}^{\mathrm{e}}$ millénaire. In P.A. de Labriffe, E. Thirault (dir.). Produire des haches au Néolithique, de la matière première à l'abandon. Paris, Société préhistorique française, p. 219-234.

Sheridan A., Pailler Y., 2012. Les haches alpines et leurs imitations en Grande Bretagne, dans l'île de Man, en Irlande et dans les îles Anglo-Normandes. In Pétrequin, Cassen et al., 2012a, p. 1046-1087.

Sheridan A., Field D., Pailler Y., Pétrequin P., Errera M., Cassen S., 2010. The Breamore jadeitite axehead and other neolithic axeheads of Alpine rock from Central Southern England. Wiltshire Archaeological and Natural History, 103, p. 16-34.

Trnka G., 2010. Die Erforschung der mittelneolithischen Kreisgrabenanlage von Kamegg im Waldviertel, Niederösterreich. In F. Bertemes, H. Meller (ed.). Neolithische Kreisgrabenanlagen in Europa. Landesamt für Denkmalpflege und Archäologie Sachsen-Anhalt, Halle, Landesmuseum für Vorgeschichte, p. 183-207.

Tsonev T., 2008. The social dimension in prehistory: a jadeite axe case in Bulgaria. In R.I. Kostov, B. Gaydarska, M. Gurova (ed.). Geoarchaeology and Archaeomineralogy. Proceedings of the International Conference, 29-30 octobre 2008. Sofia, St Ivan Rilsky, p. 60-62.

Vaquer J., Pétrequin P., 2015. La néphrite pyrénéenne. In Chancerel et al., 2015, p. 55-59.

Vaquer J., Pétrequin P., Defois B., 2011. Une hache de type Pauilhac au Grès Haut, Calvignac (Lot). Préhistoire du SudOuest, 19, 2011-2, p. 197-213.

Venturino Gambari M. (ed.), 1996. Le vie della pietra verde. L'industria litica levigata nella preistoria dell'Italia settentrionale. Torino, Omega Edizioni. 